Nada M. Al-Sayagh BDS, MSc (Asst. Prof.)

\section{The Relationship of Palatal Dimensions for Iraqi Adolescents with Different Dental Angle Classifications}

\author{
Dept of Pedod, orthod, and Prev Dentistry \\ College of Dentistry, University of Mosul
}

\begin{abstract}
Aims: To determine and compare the palatal dimensions of Iraqi adolescents with different Angle classifications in Mosul City, and to determine the correlation among the palatal dimensions. Materials and Methods: the sample consist of 142 orthodontic models of school students aged $13-17$ years with different occlusal relationships (class I normal occlusion, class II (division 1 and 2) and class III malocclusions). Eleven palatal parameters were measured by using dental vernier and included; dental arch width at the canine, 1st premolar, 2nd premolar, 1st molar, arch depth at canine and 1st molar, palatal height at canine,1st premolar, 2nd premolar, 1st molar and arch perimeter. The mean and standard deviation were calculated, analysis of variance(ANOVA), Duncan's multiple analysis range test and Pearson's correlation coefficient were used for the statistical analysis. Results: Class I normal occlusion had wider inter 1st premolar width and shallower palate at the canine ,premolars and molar region as compared with malocclusion groups in both genders, while Class II division 1had narrower and higher palate than other occlusal groups. Class II division 2 had shorter canine and molar depth than other occlusal groups in both genders in addition Class II division 2 had smaller arch perimeter as compared to other occlusal groups in females. The correlation among palatal dimensions revealed that palatal widths, arch perimeter and molar depth were highly and positively correlated with each other, while palatal height measurements were only positively correlated to each other and negatively poorly correlated to other measurements in all occlusal groups except in $\mathrm{Cl}$ II division 1 in males. This shows a highly significant correlation of palatal height at 2nd premolar and 1st molar with inter premolars and 1st molar width. Conclusions: Differences exist in most of palatal dimensions among different types of malocclusion and Class I normal occlusion these differences may help to define the dental characteristics of these problems and simplify their therapeutic managements and palatal expansion may be considered before or during treatment class II division 1.

Key words: Arch perimeters, palatal width, depth and height, malocclusion, normal occlusion.
\end{abstract}

Al-Sayagh N M. The Relationship of Palatal Dimensions for Iraqi Adolescents with Different Dental Angle Classifications. Al-Rafidain Dent J. 2011; 11(2): 251-259.

Received: 23/11/2009 Sent to Referees: 3/12/2009

Accepted for Publication: 16/3/2010

\section{INTRODUCTION}

Information regarding maxillary arch dimensions in human populations is important to orthodontics, prosthodontics, and oral surgery. It also is of interest to anthropologists and other students of human oral biology. ${ }^{(1)}$

There are several studies regarding palatal dimensions assessment, ${ }^{(2-7)}$ some of the investigators evaluating transverse dimensions had reported that maxillary arch was narrower in patient with class II division 1 malocclusion and an expansion was needed during or before treatment. ${ }^{(2-5)}$ Buschang et al. ${ }^{(8)}$ evaluated the differences in dental arch morphology among untreated adult females with class I , class II division 1 and class II division 2 malocclusion and reported that classII division 1 females had longest and narrowest arches. Johnson et al..$^{(9)}$ compared the palatal dimensions (width, length and depth) in adult occlusion and malocclusions (Cl-Icrowded, $\mathrm{Cl}$ II division1, $\mathrm{Cl}$ II division 2 and $\mathrm{Cl}$ III) in a pilot study. It was found that $\mathrm{Cl}$ II division 1 palates were narrowest in width and Cl II division 2 palate were shortest in length with other groups similar for these dimensions , $\mathrm{Cl}$ III and $\mathrm{Cl}$ I crowded subjects had the deepest palates and $\mathrm{Cl}$ II division 2 samples having the shallowed palate.

Few attempts ${ }^{(10,11)}$ were made to confirm a possible relation of dental arch dimensions. Review of literature indicated that insufficient studies evaluated palatal 
dimensions because limited number of related studies and due to the fact that studies do not include several variables describing these differences. However there are no data available about the relationships of palatal dimensions among different Angle classification groups for Iraqi adolescents in Mosul City.

The objectives of this study were: to determine and compare palatal width ,depth, height and arch perimeter in different Angle classification for Iraqi adolescents in Mosul City. Furthermore to explore if there is might be a correlation between the palatal dimensions in different occlusion groups for males and females groups.

\section{MATERIALS AND METHODS}

The samples for this study consisted of 142 Iraqi students with different dental Angle classifications. All subjects were born and living in Mosul City and were between 13 and 17 years of age. After dental classification, the distribution of the sample were as follows: class I normal occlusion (20 of each gender); class II division 1(20 of each gender); class II division 2 (16 of each gender) and class III (15 of each gender).

The inclusion criteria for the subjects were as follows: all teeth present except third molars; no significant medical history; no history of trauma, no previous orthodontic, prosthodontic treatment and no maxillofacial or plastic surgery. ${ }^{(6)}$ In addition, the class I normal occlusion had the following criteria: normal occlusion (Angle class I molar and canine relationship). Harmonious overjet and over bite (> 1and $<4)$. No crowding or spacing and no transverse discrepancies. ${ }^{(12)}$

Another inclusion criteria for the Class II groups were:

1)Bilateral Class II molar and canine relationship,

2)Absence of posterior crossbite

3)minor crowding or spacing.

Additional inclusion criteria for subsequent allocation to the Class II division 1 and Class II division 2 groups were ;

Class II division 1 group; proclination of upper front teeth with overjet $\geq 5 \mathrm{~mm}$. Class II division 2 group; retroclination of upper incisors ,at least of the two central incisors. ${ }^{(12)}$

Additional criteria used to select Class III group were: (1) bilateral Class III molar relationship in centric occlusion (2) Class III permanent canine relationship with negative overjet. ${ }^{(13)}$.

Eleven palatal measurements were recorded from each subject's maxillary dental casts using electronic digital vernia (China).and recording the data to the nearest $0.1 \mathrm{~mm}$. These measurements used in this study are as follows:

The palatal width measurements were measured as the distance between the lingual surface at the gingival margin of the right and left ; canines (ICW), 1st premolar (IP1W) , 2nd premolar (IP2W) and at the gingival margin of mesiolingual cusp of 1st molar(IMW) ${ }^{(14)}$. arch depth : The vertical distance from the interincisal midline point to the inter canine distance at the cusp tips (CD) and to the intermolar distance at the mesiolingual cusp tips (MD). ${ }^{(15)}$

Palatal height: The vertical distance at the midpalatal suture was measured by adjusting a digital depth measuring vernier between the palate and perpendicular to the plane touching the right and left cusp tips of canines( $\mathrm{PCH}$ ) ,buccal cusp tip of 1st premolars ( $\mathrm{PP} 1 \mathrm{H})$, buccal cusp tip of 2nd premolars ( $\mathrm{PP} 2 \mathrm{H})$ and mesiobuccal cusp tip of 1st molars(PMH). ${ }^{(10)}$

Arch perimeters (A P): This was measured from the distal surface of the right first molar around the arch to the distal surface of the left one over the contact points of the posterior teeth and incisal edges of the incisors by using flexible ruler.

All statistical analyses were performed using the Statistical Package for Social Sciences (SPSS for windows 98, version 11.0 SPSS Inc., Chicago). The mean and standard deviation for each variable in the different occlusion groups were calculated. Analysis of variance and Duncan's multiple range test were done for test the significance differences at $p \leq 0.05$ among different occlusion groups. Pearson's correlation were done for the palatal dimensions with each other in different occlusal categories. 


\section{RESULTS}

Palatal width: Table (1) showed no significant differences found among different occlusion groups In the ICW, IP2W, IMW while IP1W was significantly greater in class I normal occlusion group than the malocclusion groups. However there was no significant difference among the malocclusion groups in females. whereas in males also there was no significant difference in the ICW among the occlusion groups, while the class II division 1 had the narrowest IP1Wand IMW as compared with class II division 2, class III and class I normal occlusion.

Table (1): Comparison of palatal widths among different occlusion groups

\begin{tabular}{|c|c|c|c|c|c|c|c|c|c|c|c|}
\hline \multirow[b]{2}{*}{ Variable• } & \multirow[b]{2}{*}{$\begin{array}{l}\text { Occlusion } \\
\text { group }\end{array}$} & \multicolumn{5}{|c|}{$\mathrm{Ma}$} & \multicolumn{5}{|c|}{ Female } \\
\hline & & No. & mean & SD & $\begin{array}{l}P^{*} \\
\text { value }\end{array}$ & $\begin{array}{l}\text { Dun. } \\
* *\end{array}$ & No. & Mean & $\mathrm{SD}$ & $\begin{array}{l}P * \\
\text { value }\end{array}$ & $\begin{array}{l}\text { Dun. } \\
* *\end{array}$ \\
\hline \multirow{4}{*}{$\begin{array}{c}\text { Inter ca- } \\
\text { nine } \\
\text { width } \\
\text { (ICW) }\end{array}$} & CL I & 20 & 24.89 & 1.84 & \multirow{4}{*}{.368} & A & 20 & 24.57 & 1.50 & \multirow{4}{*}{.528} & $\mathrm{a}$ \\
\hline & & 20 & 83 & 2.09 & & A & 20 & 23.32 & 2.09 & & a \\
\hline & CL II2 & 16 & 24.97 & 1.70 & & A & 16 & 23.76 & 1.73 & & $\mathrm{a}$ \\
\hline & CLIII & 15 & 25.83 & 2.48 & & A & 15 & 24.53 & 2.56 & & a \\
\hline Inter $1^{\text {st }}$ & CL I & 20 & 29.98 & 1.17 & \multirow{4}{*}{.002} & B & 20 & 28.24 & 1.93 & \multirow{4}{*}{.018} & $\mathrm{~b}$ \\
\hline \multirow{3}{*}{$\begin{array}{l}\text { premolar } \\
\text { width } \\
\text { (IP1W) }\end{array}$} & $\mathrm{CL}$ & 20 & 25.42 & 2.71 & & A & 20 & 26.27 & 2.29 & & $\mathrm{a}$ \\
\hline & CL I & 16 & 28.85 & 1.59 & & $\mathrm{AB}$ & 16 & 26.24 & 1.50 & & $\mathrm{a}$ \\
\hline & CLIII & 15 & 29.50 & 2.74 & & B & 15 & 27.06 & 2.88 & & a \\
\hline Inter $2^{\text {nd }}$ & CL I & 20 & 33.97 & 1.66 & \multirow{4}{*}{.001} & D & 20 & 32.20 & 2.83 & \multirow{4}{*}{.140} & $\mathrm{a}$ \\
\hline \multirow{3}{*}{$\begin{array}{c}\text { premolar } \\
\text { width } \\
\text { (IP2W) }\end{array}$} & $\mathrm{CL}$ & 20 & 30.27 & 2.78 & & A & 20 & 30.50 & 2.45 & & a \\
\hline & $\mathrm{CL}$ & 16 & 32.25 & 2.41 & & B & 16 & 30.06 & 2.29 & & $\mathrm{a}$ \\
\hline & CLIII & 15 & 34.10 & 1.83 & & B & 15 & 33.98 & 2.51 & & a \\
\hline \multirow{4}{*}{$\begin{array}{l}\text { Inter mo- } \\
\text { lar width } \\
\text { (IMW) }\end{array}$} & CL I & 20 & 35.31 & 1.95 & \multirow{4}{*}{.004} & $\mathrm{~B}$ & 20 & 34.46 & 2.77 & \multirow{4}{*}{.224} & $\mathrm{a}$ \\
\hline & CL I & 20 & 33.12 & 3.06 & & A & 20 & 32.60 & 2.39 & & a \\
\hline & CL II2 & 16 & 34.07 & 1.89 & & $\mathrm{AB}$ & 16 & 32.28 & 2.33 & & $\mathrm{a}$ \\
\hline & CLIII & 15 & 35.63 & 2.79 & & B & 15 & 33.00 & 4.18 & & a \\
\hline
\end{tabular}

- All measurements in millimeter. ${ }^{*}$ Significant difference at $p \leq 0.05 * *$ Different letters vertically mean significant difference at $p \leq 0.05$. No.: number, Dun.: Duncan

Arch Perimeter: As shown in Table (2) , arch perimeter was significantly greater in class III than class II division 2 in female group, while in males there was no significant different among different occlusal groups.

Table (2): Comparison of arch perimeter and depth among different occlusion groups.

\begin{tabular}{|c|c|c|c|c|c|c|c|c|c|c|c|}
\hline \multirow[b]{2}{*}{ Variable } & \multirow{2}{*}{$\begin{array}{l}\text { Occlusion } \\
\text { group }\end{array}$} & \multicolumn{5}{|c|}{ Male } & \multicolumn{5}{|c|}{ Female } \\
\hline & & No. & mean & SD & $\begin{array}{l}P \\
\text { value }\end{array}$ & Dun. & No. & mean & SD & $\begin{array}{l}P \\
\text { value }\end{array}$ & Dun. \\
\hline \multirow{4}{*}{$\begin{array}{l}\text { Arch pe- } \\
\text { rimeter } \\
\quad(A P\end{array}$} & CL I & 20 & 91.60 & 3.44 & \multirow{4}{*}{.595} & $\mathrm{~A}$ & 20 & 89.79 & 3.34 & \multirow{4}{*}{.136} & $a b$ \\
\hline & CL II1 & 20 & 91.61 & 4.50 & & A & 20 & 89.13 & 4.43 & & $a b$ \\
\hline & CL II2 & 16 & 90.69 & 4.77 & & A & 16 & 86.22 & 3.29 & & $\mathrm{a}$ \\
\hline & CLIII & 15 & 90.43 & 5.38 & & A & 15 & 90.72 & 5.09 & & $\mathrm{~b}$ \\
\hline \multirow{4}{*}{$\begin{array}{c}\text { Canine } \\
\text { depth } \\
\text { (CD) }\end{array}$} & CL I & 20 & 8.69 & 1.07 & \multirow{4}{*}{.886} & B & 20 & 9.04 & .773 & \multirow{4}{*}{.000} & $\mathrm{~b}$ \\
\hline & CL II1 & 20 & 9.79 & 2.02 & & B & 20 & 9.11 & 1.47 & & $\mathrm{~b}$ \\
\hline & CL II2 & 16 & 7.32 & 1.08 & & A & 16 & 6.65 & 1.19 & & a \\
\hline & CLIII & 15 & 9.49 & 1.60 & & B & 15 & 9.25 & 1.53 & & $\mathrm{~b}$ \\
\hline \multirow{4}{*}{$\begin{array}{l}\text { Molar } \\
\text { depth } \\
\text { (MD) }\end{array}$} & CL I & 20 & 31.41 & 1.72 & \multirow{4}{*}{.003} & $\mathrm{~A}$ & 20 & 35.80 & 1.79 & \multirow{4}{*}{.000} & $\mathrm{c}$ \\
\hline & CL II1 & 20 & 33.40 & 2.36 & & B & 20 & 31.81 & 2.08 & & $\mathrm{~b}$ \\
\hline & CL II2 & 16 & 29.81 & 2.08 & & A & 16 & 28.72 & 2.01 & & $\mathrm{a}$ \\
\hline & CLIII & 15 & 30.95 & 2.41 & & A & 15 & 32.19 & 2.88 & & $\mathrm{~b}$ \\
\hline
\end{tabular}

Vertically for each variable means with the different letter are significantly different at $p \leq 0.05$ No.:number, Dun.: Duncan 
Arch depth: Canine and molar depth were significantly smaller in class II division 2 than other occlusion groups in female group, also in males, the class II division 2 had smaller canine depth than other occlusion groups while class II division 1 had larger molar depth than other occlusion groups, however there was no significant difference among other occlusion groups (Table 2).

Palatal heights: In female group as shown in Table (3), the class I normal oc- clusion had lower PCH ,PP1H and PMH than the malocclusion groups, on the other hand the class II division 2 had the higher $\mathrm{PCH}$ and PP1H.

In male group, the class II division 1 had higher $\mathrm{PP} 1 \mathrm{H}$ than other occlusion groups ,in addition class II division 1 had higher $\mathrm{PP} 2 \mathrm{H}$ and $\mathrm{PMH}$ than class I normal occlusion . on the other hand, class I had lower canine height than malocclusion groups (Table 3).

Table(3): Comparison of palatal heights among different occlusion groups.

\begin{tabular}{|c|c|c|c|c|c|c|c|c|c|c|c|}
\hline \multirow[b]{2}{*}{ Variable• } & \multirow[b]{2}{*}{$\begin{array}{l}\text { Occlusion } \\
\text { group }\end{array}$} & \multicolumn{5}{|c|}{ Male } & \multicolumn{5}{|c|}{ Female } \\
\hline & & No. & mean & $\mathrm{SD}$ & $\begin{array}{l}P \\
\text { value }\end{array}$ & Dun. & No. & mean & $\mathrm{SD}$ & $\begin{array}{l}P \\
\text { value }\end{array}$ & Dun. \\
\hline \multirow{4}{*}{$\begin{array}{c}\text { Palatal } \\
\text { Canine } \\
\text { height } \\
(\mathrm{PCH})\end{array}$} & CL I & 20 & 6.23 & 1.15 & \multirow{4}{*}{.005} & $\mathrm{~A}$ & 20 & 5.97 & 1.00 & \multirow{4}{*}{.000} & $\mathrm{a}$ \\
\hline & CL II1 & 20 & 9.37 & 1.79 & & B & 20 & 7.55 & 1.38 & & $\mathrm{~b}$ \\
\hline & CL II2 & 16 & 8.80 & 1.29 & & B & 16 & 9.13 & 1.91 & & $\mathrm{c}$ \\
\hline & CLIII & 15 & 8.45 & 1.54 & & B & 15 & 7.74 & 1.01 & & $\mathrm{~b}$ \\
\hline \multirow{4}{*}{$\begin{array}{c}\text { Palatal } 1^{\text {st }} \\
\text { premolar } \\
\text { height } \\
\text { (PP1H) }\end{array}$} & CL I & 20 & 13.77 & 2.00 & \multirow{4}{*}{.000} & $\mathrm{~A}$ & 20 & 13.05 & 1.06 & \multirow{4}{*}{.000} & $\mathrm{a}$ \\
\hline & CL II1 & 20 & 17.39 & 2.40 & & B & 20 & 14.79 & 1.59 & & $\mathrm{~b}$ \\
\hline & CL II2 & 16 & 15.12 & 2.37 & & A & 16 & 16.24 & .970 & & $\mathrm{c}$ \\
\hline & CLIII & 15 & 13.85 & 1.90 & & $\mathrm{~A}$ & 15 & 13.86 & 1.91 & & $a b$ \\
\hline \multirow{4}{*}{$\begin{array}{c}\text { Palatal } 2^{\text {nd }} \\
\text { premolar } \\
\text { height } \\
\text { (PP2H }\end{array}$} & CL I & 20 & 17.49 & 2.38 & \multirow{4}{*}{.000} & $\mathrm{~A}$ & 20 & 15.89 & 1.18 & \multirow{4}{*}{.000} & $\mathrm{a}$ \\
\hline & CL II1 & 20 & 20.96 & 2.18 & & B & 20 & 18.48 & 2.12 & & $\mathrm{~b}$ \\
\hline & CL II2 & 16 & 19.18 & 1.92 & & $\mathrm{AB}$ & 16 & 19.13 & 1.27 & & $\mathrm{~b}$ \\
\hline & CLIII & 15 & 18.69 & 2.19 & & $\mathrm{~A}$ & 15 & 18.17 & 2.20 & & $\mathrm{~b}$ \\
\hline \multirow{4}{*}{$\begin{array}{c}\text { Palatal } \\
\text { molar } \\
\text { height } \\
\text { (PMH }\end{array}$} & CL I & 20 & 17.98 & 2.39 & \multirow{4}{*}{.001} & $\mathrm{~A}$ & 20 & 16.72 & 1.33 & \multirow{4}{*}{.000} & $\mathrm{a}$ \\
\hline & CL II1 & 20 & 20.65 & 2.47 & & B & 20 & 19.04 & 1.69 & & b \\
\hline & CL II2 & 16 & 19.94 & 1.78 & & $\mathrm{AB}$ & 16 & 19.52 & 2.27 & & $\mathrm{~b}$ \\
\hline & CLIII & 15 & 19.39 & 3.59 & & $\mathrm{AB}$ & 15 & 20.47 & 2.10 & & b \\
\hline
\end{tabular}

Vertically for each variable means with the different letter are significantly different at $p \leq 0.05$

The correlation among palatal widths, depth, height and arch perimeter in different occlusion groups for males and females are shown in Tables (4) and (5) respectively. Some of which showed highly significant correlation while others showed weak relationship.
Generally, in both gender palatal width, molar depth and arch perimeter were positively correlated to each other, while palatal height measurements were only positively correlated to each other and negatively correlated to other measurements. 
Table (4): Correlation between palatal dimensions in different occlusion groups in males .

\begin{tabular}{|c|c|c|c|c|c|c|c|c|c|c|c|}
\hline $\begin{array}{l}\text { vari- } \\
\text { able }\end{array}$ & $\begin{array}{l}\text { Occlu- } \\
\text { sion } \\
\text { group }\end{array}$ & ICW & $\mathbf{I P} \mathbf{P}_{\mathbf{1}} \mathbf{W}$ & $\mathbf{I P}_{\mathbf{2}} \mathbf{W}$ & IMW & $\mathbf{A P}$ & CD & MD & PCH & $\mathbf{P} \mathbf{P}_{\mathbf{1}} \mathbf{H}$ & $\mathbf{P P} \mathbf{P}_{2} \mathbf{H}$ \\
\hline \multirow{4}{*}{$\mathbf{I P}_{\mathbf{1}} \mathbf{W}$} & CL I & $.609 *$ & & & & & & & & & \\
\hline & CLII1 & $.717 * *$ & & & & & & & & & \\
\hline & CLII2 & $.620^{*}$ & & & & & & & & & \\
\hline & CLIII & $.850 *$ & & & & & & & & & \\
\hline \multirow{4}{*}{$\mathbf{I P}_{\mathbf{2}} \mathbf{W}$} & CL I & $.339 *$ & $.757 * *$ & & & & & & & & \\
\hline & CLII1 & $.576^{*}$ & $.906 * *$ & & & & & & & & \\
\hline & CLII2 & $.703^{*}$ & $.856 * *$ & & & & & & & & \\
\hline & CLIII & $.926 * *$ & $.980^{* *}$ & & & & & & & & \\
\hline \multirow{4}{*}{ IMW } & CL I & .131 & $.649 * *$ & $.880^{* *}$ & & & & & & & \\
\hline & CLII1 & $.689 * *$ & $.853^{* *}$ & $.916^{* *}$ & & & & & & & \\
\hline & CLII2 & .635 & $.708 *$ & $.760 *$ & & & & & & & \\
\hline & CLIII & $\begin{array}{l}-.010 \\
\end{array}$ & .472 & .306 & & & & & & & \\
\hline \multirow{4}{*}{$\mathbf{A P}$} & CL I & $.703 * *$ & $.538^{*}$ & $.538^{*}$ & .394 & & & & & & \\
\hline & CLII1 & $.720 * *$ & $.558^{*}$ & $.541 *$ & $.697^{* *}$ & & & & & & \\
\hline & CLII2 & $.920 * *$ & .652 & $.767^{*}$ & $.711^{*}$ & & & & & & \\
\hline & CLIII & .306 & $.726^{*}$ & .599 & $.893^{*}$ & & & & & & \\
\hline \multirow{4}{*}{ CD } & CL I & .238 & .066 & .227 & .017 & .506 & & & & & \\
\hline & CLIII & .115 & -.127 & -.120 & .010 & $.553^{*}$ & & & & & \\
\hline & CLII2 & .490 & -.038 & .190 & .030 & .486 & & & & & \\
\hline & CLIII & $-.814 *$ & $\begin{array}{l}-.629 \\
\end{array}$ & $\begin{array}{l}-.740 \\
\end{array}$ & .026 & -.208 & & & & & \\
\hline \multirow{4}{*}{ MD } & CL I & $.523^{*}$ & .221 & .323 & .067 & $.864 * *$ & $.746^{*}$ & & & & \\
\hline & CLII1 & .478 & .239 & .217 & .350 & $.866^{* *}$ & $.774 * *$ & & & & \\
\hline & CLII2 & $.921 * *$ & .514 & $.695^{*}$ & .528 & $.945^{* * *}$ & $.702 *$ & & & & \\
\hline & CLIII & .023 & .494 & .340 & .805 & $.903^{*}$ & .171 & & & & \\
\hline \multirow{4}{*}{ PCH } & CL I & .051 & .076 & .272 & .205 & .321 & $.587 *$ & .424 & & & \\
\hline & CLII1 & -.402 & -.413 & -.260 & -.364 & -.024 & .314 & .164 & & & \\
\hline & CLII2 & .613 & .546 & $.684 *$ & .359 & $.734^{*}$ & $.726^{*}$ & $.797^{*}$ & & & \\
\hline & CLIII & -.191 & .077 & .041 & .122 & .073 & .290 & .237 & & & \\
\hline \multirow{4}{*}{$\mathbf{P P}_{1} \mathbf{H}$} & CL I & .252 & .021 & .109 & -.052 & .378 & $.596^{*}$ & .448 & $.836^{* * *}$ & & \\
\hline & CLII1 & $\begin{array}{l}-.339 \\
\end{array}$ & -.491 & -.411 & -.474 & -.109 & .200 & .024 & $.591 *$ & & \\
\hline & CLII2 & -.341 & .166 & .036 & .088 & -.195 & .028 & -.253 & .231 & & \\
\hline & CLIII & $\begin{array}{l}-.323 \\
\end{array}$ & -.040 & -.137 & .158 & .050 & .675 & .331 & $.816^{*}$ & & \\
\hline \multirow{4}{*}{$\mathbf{P P}_{2} \mathbf{H}$} & CL I & $.541^{*}$ & .045 & .081 & -.102 & .444 & .447 & .498 & $.521^{*}$ & $.852 * *$ & \\
\hline & CLII1 & -.375 & $-.702 * *$ & $-.689^{* *}$ & $-.680 * *$ & -.390 & -.075 & -.185 & .420 & $.746^{* *}$ & \\
\hline & CLII2 & .393 & .526 & .548 & .600 & .517 & .524 & .487 & $.752^{*}$ & .582 & \\
\hline & CLIII & .020 & .149 & .139 & $\begin{array}{l}-.053 \\
\end{array}$ & -.105 & .233 & .005 & $.893^{*}$ & $.831^{*}$ & \\
\hline \multirow{4}{*}{ PMH } & CL I & $.697 * *$ & .265 & .250 & .164 & .473 & .207 & .315 & .346 & $.617^{*}$ & $.854 * *$ \\
\hline & CLII1 & -.478 & $-.726^{* *}$ & $-.702 * *$ & $-.672 * *$ & -.464 & -.186 & -.223 & .338 & .498 & $.912 * *$ \\
\hline & CLII2 & .493 & .444 & .554 & .504 & .535 & .665 & .581 & $.798 * *$ & .350 & $.933 * *$ \\
\hline & CLIII & .016 & .192 & .177 & -.051 & .031 & .274 & .236 & $.918^{* *}$ & $.846^{*}$ & $.912 * *$ \\
\hline
\end{tabular}


Table (5): Correlation between palatal dimensions in different occlusion groups in females.

\begin{tabular}{|c|c|c|c|c|c|c|c|c|c|c|c|}
\hline $\begin{array}{l}\text { vari- } \\
\text { able }\end{array}$ & $\begin{array}{l}\text { Occlu- } \\
\text { sion } \\
\text { group }\end{array}$ & ICW & $\mathbf{I P}_{1} \mathbf{W}$ & $\mathbf{I P}_{\mathbf{2}} \mathbf{W}$ & IMW & $\mathbf{A P}$ & CD & MD & PCH & $\mathbf{P P}_{1} \mathbf{H}$ & $\mathbf{P P}_{2} \mathbf{H}$ \\
\hline \multirow{4}{*}{$\mathbf{I P}_{1} \mathbf{W}$} & CL I & $.596^{*}$ & & & & & & & & & \\
\hline & CLII1 & $.701 * *$ & & & & & & & & & \\
\hline & CLII2 & .272 & & & & & & & & & \\
\hline & CLIII & $.761 *$ & & & & & & & & & \\
\hline \multirow{4}{*}{$\mathbf{I P}_{\mathbf{2}} \mathbf{W}$} & CL I & $.670 * *$ & $.721 * *$ & & & & & & & & \\
\hline & CLII1 & $.637 *$ & $.935^{* *}$ & & & & & & & & \\
\hline & CLII2 & .563 & $.710^{*}$ & & & & & & & & \\
\hline & CLIII & $.745^{*}$ & .588 & & & & & & & & \\
\hline \multirow{4}{*}{ IMW } & CL I & $.736 * *$ & $.706^{* *}$ & $.923 * *$ & & & & & & & \\
\hline & CLII1 & .479 & $.873 * *$ & $.894 * *$ & & & & & & & \\
\hline & CLII2 & .451 & 575 & $.969 * *$ & & & & & & & \\
\hline & CLIII & $.700 *$ & .431 & $.918 * *$ & & & & & & & \\
\hline \multirow{4}{*}{ AP } & CL I & $.929 * *$ & .380 & $.545^{*}$ & $.637 *$ & & & & & & \\
\hline & CLII1 & .532 & $.545 *$ & $.563^{*}$ & $.573 *$ & & & & & & \\
\hline & CLII2 & .565 & $.783 *$ & $.881 * *$ & $.830^{*}$ & & & & & & \\
\hline & CLIII & $.708 *$ & .588 & .622 & $.754 *$ & & & & & & \\
\hline \multirow{4}{*}{ CD } & CL I & .348 & -.097 & -.219 & -.093 & $.553^{*}$ & & & & & \\
\hline & CLII1 & -074 & -.216 & $\begin{array}{l}.099 \\
\end{array}$ & -.224 & $\begin{array}{l}-.068 \\
\end{array}$ & & & & & \\
\hline & CLII2 & -.192 & .651 & .238 & .185 & .479 & & & & & \\
\hline & CLIII & .354 & .615 & .130 & .227 & .461 & & & & & \\
\hline \multirow{4}{*}{ MD } & CL I & $.637 *$ & .111 & .076 & .145 & $.810 * *$ & $.861 * *$ & & & & \\
\hline & CLII1 & .186 & .091 & .162 & .093 & .312 & $.678 * *$ & & & & \\
\hline & CLII2 & .015 & .688 & .456 & .415 & .697 & $.900 * *$ & & & & \\
\hline & CLIII & .402 & .510 & .403 & .590 & .811 & .266 & & & & \\
\hline \multirow{4}{*}{ PCH } & CL I & -.091 & -.014 & -.100 & -.069 & -.126 & -.099 & -.181 & & & \\
\hline & CLII1 & .038 & -146 & -.030 & $\begin{array}{l}-.023 \\
\end{array}$ & -.465 & -.204 & -.463 & & & \\
\hline & CLII2 & -.001 & -.290 & -.153 & -.170 & -.178 & .148 & .138 & & & \\
\hline & CLIII & -.548 & -.311 & -.606 & -.373 & -.219 & .449 & .266 & & & \\
\hline \multirow{4}{*}{$\mathbf{P} \mathbf{P}_{1} \mathbf{H}$} & CL I & .073 & .192 & .060 & -.082 & .021 & .043 & .054 & $.697 * *$ & & \\
\hline & CLII1 & .236 & $\begin{array}{l}.097 \\
\end{array}$ & .253 & .202 & -.196 & .088 & -.246 & $.854 * *$ & & \\
\hline & CLII2 & -.283 & -.232 & -.170 & -.158 & -.291 & .069 & -.110 & $.741 *$ & & \\
\hline & CLIII & -.533 & -.281 & -.264 & $\begin{array}{l}-.099 \\
\end{array}$ & $\begin{array}{l}-.291 \\
\end{array}$ & .039 & .150 & .328 & & \\
\hline \multirow{4}{*}{$\mathbf{P P}_{2} \mathbf{H}$} & CL I & -.044 & .059 & .080 & -.152 & -.074 & -.012 & -.068 & .282 & $.712 * *$ & \\
\hline & CLII1 & .493 & .244 & .183 & .317 & .357 & .282 & -.202 & .355 & .346 & \\
\hline & CLII2 & -104 & .146 & -.065 & -.097 & .162 & .118 & .110 & .022 & .350 & \\
\hline & CLIII & -.322 & -.235 & -.203 & -.087 & .257 & -.108 & .222 & .234 & $.799 *$ & \\
\hline \multirow{4}{*}{ PMH } & CL I & .068 & -.013 & .244 & .176 & .194 & -.109 & .102 & -.009 & .090 & .095 \\
\hline & CLII1 & .318 & .211 & .359 & .332 & .083 & -.151 & -.193 & $.606 *$ & $.641 *$ & .260 \\
\hline & CLII2 & -.104 & .253 & .046 & .058 & .376 & .307 & .357 & -.163 & .060 & $.903 * *$ \\
\hline & CLIII & .088 & .014 & -.228 & -.102 & .499 & .053 & .290 & .016 & .346 & $.774 *$ \\
\hline
\end{tabular}

\section{DISCUSSION}

Palatal dimensions were investigated in a few of the previous studies, and conflicting results were found. These differences may be due to the several factors ; gender dimorphism, ethnic and racial differences, sample selection or severity of malocclusion and size and age of the subjects examined. ${ }^{(16,17)}$

Inter canine width in the present study showed no significant difference between class I normal occlusion and malocclusion groups in both genders. This is in agreement with Bishara et $a l^{(2)}$, Sayin 
and Turkkahraman ${ }^{(5)}$ who reported that no difference in the inter canine width between class I and class II division 1 group and also in agreement with Al-khateeb et $a l .{ }^{(18)}$ who found no significant difference between $\mathrm{Cl}$ II division 1 and $\mathrm{Cl}$ II division 2 , but in contrast to staley et al. ${ }^{(4)}$ Who reported that adults with normal occlusion had larger maxillary canine width than the malocclusion subjects this may be attributed to difference in the age. Furthermore, the finding of the present study showed that there was no significant difference among different occlusion groups in female group in the ICW, IP2Wand IMW,thus these measurements were not dependent factors in classification of different occlusion groups in females group.

The results of males in the present study revealed that class II division 1 had smaller IP1W,IP2W and IMW than other occlusion groups. This is in agreement with Staley et $a l^{(4)}$ and Sayin and Turkkahraman ${ }^{(5)}$ who suggested that the narrow widths of the dental arch in Class II division 1 appeared to be caused by palatally tipped teeth and also by narrower bony bases of the dental arch, therefore they concluded that slow maxillary expansion may be considered before or during the treatment of class II division 1. However, in contrast with previous studies, the findings of Uysal ${ }^{(6)}$ indicated that the upper intermolar widths were larger in patients with Class II division 1 when compared with the normal occlusion. Therefore, they concluded that subjects with Class II division 1 tend to have the maxillary molar teeth inclined to the buccal to compensate for the insufficient alveolar base. For that reason, rapid maxillary expansion rather than slow expansion may be considered.

.Lux $e t_{a l}{ }^{(12)}$ found that the inter molar widths were about $3-5 \mathrm{~mm}$ smaller in the class II division 1 group than class I and good occlusion groups. Lux et al. ${ }^{(12)}$ also reported that first molar width in class II division 2 cases took up a position between the class II division 1 and class I control group. These results support the findings in the present study for males. Clinicians have speculated that nasal obstruction, finger habits, tongue thrusting, low tongue position, and abnormal swal- lowing and sucking behaviors were reasons for narrower maxillary dental arch widths in Class II division 1 malocclusions compared with a normal occlusion sample. (6)

Although in this study, the class III in female group had smaller ICW, IP2W and IMW than class I normal occlusion but these differences are not statistically significant however IP1W was significantly smaller in class III. This is in accordance with Ysal et al. ${ }^{(13)}$ who found that the maxillary interpremolar, intermolar width were significantly narrower in class III group than in class I normal occlusion.

Lingually positioned maxillary posterior crossbites are often seen in the Class III malocclusion. One could speculate that during eruption in Class III subjects, the maxillary posterior teeth compensate for the buccal relationships (that result from the anteroposterior displacement of the jaws) by palatal movement to avoid inappropriate contacts with the lower teeth. Besides, it was widely believed that a wide and big mandible obstructed growth and development of the maxillary dental and alveolar arches.

The smaller maxillary arch perimeter and depth in class II division 2 as compared with other occlusion groups may be explained by the palatal inclination of upper central incisors, where as the larger molar depth in the class II division 1 for male group may be explain by labial inclination of the upper central incisors ,this support the finding of Papagerogiou et $a l .{ }^{(19)}$ The results of this study showed that the class II division lin male group had the deepest palate while the class I normal occlusion in both genders had the shallowest palate this is in contrast to Johnson et $a l^{(9)}$ who reported that class III and class I crowded subjects had a deepest palate and class II division 2 sample had a shallowest palate and also in contrast to the finding of Zarringhalan ${ }^{(20)}$ who found that palatal height in class III was more than class I , class II/1and normal occlusion group.

The correlation revealed that palatal height were only correlated to each other ,while these parameters were not significantly correlated to any other cast measurements .the independence of the palatal height with palatal width and arch depth 
reported in this study in all occlusal groups except $\mathrm{Cl}$ II division 1 showed notable agreement with those of Kaddah ${ }^{(11)}$ and Williams, who noted separate genetic control for the palatal vault depth. On the other hand ,contradiction existed with the Eid et al. ${ }^{(10)}$ who they reported correlation between the dental arch width and the corresponding palatal vault depth. This disagreement could be attributed to differences of the selected sample in each study. Their sample was randomly selected and was restricted only to a group of children ranging in age from 9-12 year. The class II division 1 which showed strong negative correlation of $\mathrm{P} 2 \mathrm{H}, \mathrm{PMH}$ with IP1W,IP2W,IMW.this is in agreement with Eid et al. ${ }^{(10)}$

The maxillary arch perimeter was positively significant correlation with most palatal widths particularly the IMW in all occlusion groups and in both genders. On the other hand, the arch perimeter was poorly correlated to the palatal height, these results suggested that an increase in the arch perimeter was accompanied by an increase in the palatal widths especially at the inter molar width and by unchanged palatal height. this support the finding s of Eid et al. ${ }^{(10)}$ in addition arch perimeter was highly significant correlation with molar depth in all occlusal groups in male group. this indicate that increase in molar depth was accompanied by increase in arch perimeter. Paulino et al. ${ }^{(21)}$ found a very high correlation between ICW and arch length relationship. This support the finding of the present study in class II division 2 in males and class I normal occlusion in females.

\section{CONCLUSIONS}

1.Class I normal occlusion had wider inter 1st premolar width and shallower palate at the canine, premolars and molar region as compared with malocclusion groups in both genders.

2. Class II division 1 had narrower and higher palate than other occlusal groups. While Class II division 2 had shorter canine and molar depth than other occlusal groups in both genders in addition Class II division 2 had smaller arch perimeter as compared to other occlusal groups in females.
3.The correlation among palatal dimensions revealed that palatal widths, arch perimeter and molar depth were highly and positively correlated with each other. While palatal height measurements were only positively correlated to each other and negatively poorly correlated to other measurements in all occlusal groups except in $\mathrm{Cl}$ II division 1 in males. They showed a highly significant correlation of palatal height at 2nd premolar and 1st molar with inter premolars and 1st molar width.

\section{REFERENCES}

1. Younis SAES. Maxillary arch dimensions in Saudi and Egypt population sample. Am J Orthod Dentofacial Orthop. 1984; 85: 83-88.

2. Bishara SE, Bayati P, Jakobsen JR. Longitudinal comparisons of dental arch changes in normal and untreated Class II Division 1 subjects and their clinical implications. Am J Orthod Dentofacial Orthop. 1996 Nov; 110: 5483-489.

3. McNamara JA Jr. Early intervention in the transverse dimension: is it worth the effort? Am J Orthod Dentofacial Orthop.2002; 121: 572-574.

4. Staley RN, Stuntz WR, Peterson LC. A comparison of arch widths in adults with normal occlusion and adults with Class II division 1 malocclusion. Am J Orthod. 1985; 88: 163-169.

5. Sayin MO, Turkkahraman H. Comparison of dental arch and alveolar widths of patients with Class II division 1 malocclusion and subjects with Class I ideal occlusion. Angle Orthod. 2004; 74: 356-360.

6. Uysal T, Memili B, Usumez S, Sari Z. Dental and alveolar arch widthsin normal occlusion, Class II division 1 and Class II division 2. Angle Orthod.2005; 75: 941-947.

7. Nie Q, Lin J. A comparison of dental arch forms between Class II division 1 and normal occlusion assessed by Euclidean distance matrixanalysis. Am J Orthod Dentofacial Orthop. 2006; 129:528-535.

8. Buschang PH, Stroud J, Alexander RG. Differences in dental archmorphology among adult females with untreated 
Class I and Class II malocclusion. Eur J Orthod. 1994;16:47-52.

9. Johnson JG ,Kuntz TR, Staley RN, Jakobsen JR. Comparison of palatal dimensions in adult normal occlusion and malocclusion. J Dent Res. 1994:73.

10.Eid AA, El-Nammrawy MM, Kadry WA. The relationship between width, depth and circumference of dental arch for group of Egyptian school children. Egypt J Orthod. 1987; 1(2): 113-137.

11.Kaddah MAF.A cluster analysis of study cast measurements for agroup of Egyptian adults having normal occlusion. Cario Denl J. 1998; 14(2): 283292.

12. Lux CJ, Conradt C, Burden D, Komposch G. Dental arch widths and mandibular-maxillary base widths in Class II malocclusion betweenearly mixed and permanent dentitions. Angle Orthod. 2003; 73: 674-685.

13.Uysal T, Usumez S, Memili B, Sari Z. Dental and alveolar arch widths in normal occlusion, and Class III malocclusion. Angle Orthod. 2004; 75(5): 809-813.

14.McNamara JA, Baccetti T, Franchi L, Herberger TA. Rapid maxillary expansion followed by fixed appliances: A long -term evaluation of changes in arch dimensions. Angle Orthod. 2003;
73: 344-353.

15.Mohammad IS. Maxillary arch dimensions. A cross sectional study between 9-17 years. Master thesis, Baghdad University-Iraq;1993.

16.Huth J, Staley RN, Jacobs R, Bigelow $\mathrm{H}$, Jakobsen J. Arch width in classII-2 adults compared to adults with class II1 and normal occlusion. Angle Orthod. 2006;77(5): 837-844.

17. Walkow TM, Peck S. Dental arch width in Class II Division 2 deep-bite malocclusion. Am J Orthod Dentofacial Orthop. 2002; 122: 608-613.

18. Al -khateeb SN, Abu Alhaija ES. Tooth size discrepancies and arch parameters among different malocclusion in a Jordanian sample. Angle Orthod. 2006; 76(3): 459 - 465.

19. Papagorgiou IS, Papadopoulos MA, Zafiriadis A. Dentoalveolar characteristics in Class II,division 2 malocclusion. Hellenic Orthodontic Review. 1998; 1: 117-134.

20. Zarringhalam M. Measuring palatal height in normal occlusion and malocclusion. J Dent TUMS. 2004; 1(4): 3942.

21. Paulino V, Paredes V, Gandia JL, Cibrian R. Prediction of arch length based on inter canine width. Eur J Orthod. 2008; 30(3): 295-298. 